\title{
Some string cosmological models in Bianchi type III space-time with magnetic field and vacuum energy density
}

\author{
Raj Bali and Subhash C. Bola
}

\begin{abstract}
Bianchi type III string cosmological models (massive and geometric) in presence of magnetic field with vacuum energy density following the techniques used by Letelier (1979) and Stachel (1980) are obtained. We find that the energy conditions (dominant as well as weak) given by Hawking and Ellis (1974) are satisfied for the models. Also the solutions obtained satisfy conservation equation for massive string with magnetic field and vacuum energy density. The models in general, represent anisotropic phase of space-time due to the presence of string. However, the models isotropize at late time and in special case. It has also been shown that the magnetic field is directly linked with the matter. For string dust model, the state finder parameters $\{r, s\}$ are in excellent agreement with Planck results (Sahni et al. (2003)). The physical and geometrical aspects of the models with special cases are also discussed.
\end{abstract}

Keywords String, Cosmological, Bianchi Type III, Magnetic Field, Vacuum Energy Density.

\section{Introduction}

Bianchi Type I space-time is a very special subset of spatially homogeneous cosmological models. We, therefore, consider more general Bianchi Type III space-time for the study of string cosmological models in presence of magnetic field with vacuum energy density. Cosmic strings have received a considerable interest in cosmology because it is believed that these strings give rise to density perturbation leading to the formation of galaxies (Zel'dovich (1980)). In the early universe (string dominated era), the strings produce fluctuations in the density of particles and we may speculate that as the strings vanish then particles become important. Accordingly models of universe which evolves from string dominated era and ends up in a particle dominated era, are of physical interest. These strings have stress energy and classified as massive and geometric strings. The presence of strings in the early universe have been explained by Kibble (1976), Everet (1981) and Vilenkin (1982) using grand unified field theories. The pioneering work in the formulation of energy-momentum tensor for massive string is due to Letelier (1979) who explained that massive strings are formed by geometric strings (Stachel (1980)) with particles attached along its extension. Using this idea, Letelier (1983) obtained cosmological solutions for massive strings in the frame work of Bianchi Type I and Kantowski-Sachs space time. The magnetic field plays a significant role at the cosmological scale and is present in galactic and intergalactic spaces. The present day magnitude of the magnetic energy is very small in comparison with the estimated matter density. It might not have been negligible during early stage of the evolution of the universe. A cosmological model which contains a global magnetic field is necessarily anisotropic since the

Raj Bali and Subhash C. Bola, Department of Mathematics, University of Rajasthan, Jaipur - 302004, India. Corresponding Author: Raj Bali (e-mail: rbalijpr@gmail.com) 
magnetic field vector specifies a preferred spatial direction. Bronnikov et al. (2004) have studied the evolution of Bianchi Type I space-time with a global unidirectional electromagnetic field. Melvin (1975) in the cosmological solutions for dust and electro-magnetic field has argued that for a large part of the history of evolution of the universe, the matter was in a highly ionized state and is smoothly coupled with the field and subsequently forms a neutral matter as a result of universe expansion. Hence the presence of magnetic field in string dust universe is not unrealistic. Therefore, the string cosmological models with magnetic field have been investigated by many authors viz. Banerjee et al. (1990), Singh and Singh (1999), Chakraborty and Chakraborty (2001), Bali and Anjali (2006), Bali and Jain (2007) in different contexts. Tikekar and Patel (1992), Wang (2006) obtained some exact solutions of string cosmology in Bianchi III space-time in the presence of magnetic field. Bianchi Type III massive string cosmological models in the presence of magnetic field for perfect fluid distribution have been investigated by Bali et al. (2007, 2008) without vacuum energy density.

The cosmological constant has played a stimulating role in gravitation theory ever since. In 1917, Einstein introduced it to provide the gravitational repulsion needed to support a static universe. When Hubble's observations established the expanding nature of universe, Einstein conceded that there was no special need for $\Lambda$-term into his field equation. A new sight emerged in 1934 when Lemaitre interpreted $\Lambda$-term as Lorentz-invariant vacuum fluid in Einstein field equation. Zel'dovich (1968) in his most innovative way revived the issue of the cosmological constant $(\Lambda)$ by identifying it with the vacuum energy density due to quantum fluctuations. Therefore, cosmologists started thinking about $\Lambda$ once again with a new outlook. Before 1998, there was no direct astronomical evidence for cosmological constant $\Lambda$. In 1998, 1999 two independent groups led by Riess et al. (1998), Perlmutter et al. (1999) used Type Ia Supernovae to show that the universe is accelerating. This remarkable discovery provided the first direct evidence that $\Lambda$ is non-zero with $\Lambda \sim 1.7 \times 10^{-121}$ Planck units. It is now commonly believed by scientific community that via the cosmological constant (a kind of repulsive pressure, dubbed as dark energy) is the most suitable candidate to explain recent observations that universe is not only expanding but also accelerating. There are many attempts to understand the nature of dark energy. Astier et al. (2007) has shown that dark energy behaves like the cosmological constant to a precision of $10 \% . \Lambda \mathrm{CDM}$ models agree closely with almost all the established cosmological abbreviations. Cosmological models with time dependent $\Lambda$ term have been investigated by many researchers viz. Bertolami (1986), Berman et al. (1990), Chen and Wu (1990), Beesham (1993), Wang and Meng (2005), Saha (2007, 2013), Bali and Singh (2008, 2014).

In this paper, we have investigated some Bianchi Type III string cosmological models in the presence of magnetic field and vacuum energy density following the techniques used by Letelier(1983) and Stachel (1980). To get the deterministic model, we assume that the shear $(\sigma)$ in proportional to expansion $(\theta)$ 
and vacuum energy density $\Lambda \simeq \frac{\mathrm{a}}{\mathrm{R}^{3}}$ where $\mathrm{R}$ is scale factor and a is the constant. We find that the model represents shearing universe with singularity at $\tau=0$ and undergoes expansion. The models, in general, represent anisotropic phase of universe due to the presence of string. However, the models isotropize at late time. In special case it leads to FRW dust universe with zero curvature. The model also represents accelerating and decelerating phases of universe which matches with the result of recent astronomical observations.

\section{Metric and field equations}

We consider Bianchi Type III space-time in the form as

$$
\mathrm{ds}^{2}=-\mathrm{dt}^{2}+\mathrm{A}^{2} \mathrm{dx}^{2}+\mathrm{B}^{2} \mathrm{e}^{-2 \alpha x} \mathrm{dy}^{2}+\mathrm{C}^{2} \mathrm{dz}^{2}
$$

where $\mathrm{A}, \mathrm{B}$ and $\mathrm{C}$ are functions of ' $\mathrm{t}$ ' only. If $\alpha=0$ then the space-time leads to Bianchi Type I space-time.

The energy-momentum tensor for the string with magnetic field is taken as

$$
\mathrm{T}_{\mathrm{i}}^{\mathrm{j}}=\rho \mathrm{v}_{\mathrm{i}} \mathrm{v}^{\mathrm{j}}-\lambda \mathrm{x}_{\mathrm{i}} \mathrm{x}^{\mathrm{j}}+\mathrm{E}_{\mathrm{i}}^{\mathrm{j}}
$$

For massive string (Letelier [7])

$$
\rho=\rho_{p}+\lambda
$$

with

$$
v_{i} v^{i}=-x_{i} x^{i}=-1
$$

and

$$
\mathrm{v}^{\mathrm{i}} \mathrm{x}_{\mathrm{i}}=0
$$

with $x_{1}=0=x_{2}, x_{3} \neq 0 . \rho$ being the rest energy density of the system of strings, $\lambda$ the tension density of the strings, $\rho_{p}$ the particle density, $v^{i}$ the four-velocity and $\mathrm{x}^{\mathrm{i}}$ the direction of strings i.e. string direction is along $\mathrm{z}$-axis. Here $\mathrm{E}_{\mathrm{ij}}$ is the electromagnetic field given as

$$
\mathrm{E}_{\mathrm{ij}}=\frac{1}{4 \pi}\left[\mathrm{g}^{\ell \mathrm{m}} \mathrm{F}_{\mathrm{i} \ell} \mathrm{F}_{\mathrm{jm}}-\frac{1}{4} \mathrm{~F}_{\ell \mathrm{m}} \mathrm{F}^{\ell \mathrm{m}} \mathrm{g}_{\mathrm{ij}}\right]
$$

We assume the coordinates to be comoving so that

$$
\mathrm{v}^{1}=0=\mathrm{v}^{2}=\mathrm{v}^{3}, \mathrm{v}^{4}=1
$$

If particle density $\rho_{p}=0$ then equation (3) leads to

$$
\rho=\lambda
$$

We also assume that the current is flowing along z-axis so the magnetic field is in the xy-plane. Thus $F_{12}$ is the only non-vanishing component of electromagnetic field tensor $\left(\mathrm{F}_{\mathrm{ij}}\right)$. A cosmological model which contains a global magnetic field is 
necessarily anisotropic since the magnetic field specifies a preferred spatial direction. (Bronnikov et al.(2004)). Now the Maxwell's equations

$$
F_{i j ; k}+F_{j k ; i}+F_{k i ; j}=0 \text { and }\left(F^{i k} \sqrt{-g}\right)_{; k}=0
$$

lead to

$$
\mathrm{F}_{12}=\mathrm{Ke}^{-\alpha \mathrm{x}}
$$

where $\mathrm{K}$ and $\alpha$ are constants. Now the non-vanishing components of $\mathrm{E}_{\mathrm{ij}}$ corresponding to the metric (1) are given by

$$
\begin{aligned}
& \mathrm{E}_{11}=\frac{1}{4 \pi}\left[\mathrm{g}^{\ell \mathrm{m}} \mathrm{F}_{1 \ell} \mathrm{F}_{1 \mathrm{~m}}-\frac{1}{4} \mathrm{~F}_{\ell \mathrm{m}} \mathrm{F}^{\ell \mathrm{m}} \mathrm{g}_{11}\right] \\
& =\frac{1}{4 \pi}\left[\mathrm{g}^{22} \mathrm{~F}_{12}^{2}-\frac{1}{2} \mathrm{~F}_{12} \mathrm{~F}^{12} \mathrm{~g}_{11}\right] \\
& =\frac{1}{4 \pi}\left[\frac{\mathrm{K}^{2}}{\mathrm{~B}^{2}}-\frac{\mathrm{K}^{2}}{2 \mathrm{~B}^{2}}\right]
\end{aligned}
$$

i.e.

$$
\mathrm{E}_{11}=\frac{\mathrm{K}^{2}}{8 \pi \mathrm{B}^{2}}
$$

Thus

$$
\begin{aligned}
& E_{1}^{1}=g^{11} E_{11} \\
& =\frac{K^{2}}{8 \pi A^{2} B^{2}}
\end{aligned}
$$

and

$$
\begin{aligned}
& \mathrm{E}_{22}=\frac{1}{4 \pi}\left[\mathrm{g}^{11} \mathrm{~F}_{21} \mathrm{~F}_{21}-\frac{2}{4} \mathrm{~F}_{12} \mathrm{~F}^{12} \mathrm{~g}_{22}\right] \\
& =\frac{1}{4 \pi}\left[\frac{\mathrm{F}_{12}^{2}}{\mathrm{~A}^{2}}-\frac{1}{2} \mathrm{~g}^{11} \mathrm{~g}^{22} \mathrm{~F}_{12}^{2} \mathrm{~g}_{22}\right] \\
& =\frac{\mathrm{F}_{12}^{2}}{8 \pi \mathrm{A}^{2}}
\end{aligned}
$$

Thus

$$
\begin{aligned}
E_{2}^{2} & =g^{22} E_{22} \\
& =\frac{K^{2}}{8 \pi A^{2} B^{2}}
\end{aligned}
$$


Similarly

$$
\begin{aligned}
& \mathrm{E}_{3}^{3}=-\frac{\mathrm{K}^{2}}{8 \pi \mathrm{A}^{2} \mathrm{~B}^{2}} \\
& \mathrm{E}_{4}^{4}=-\frac{\mathrm{K}^{2}}{8 \pi \mathrm{A}^{2} \mathrm{~B}^{2}} \\
& \mathrm{E}_{1}^{4}=0
\end{aligned}
$$

The non-vanishing components of energy momentum tensor $\mathrm{T}_{\mathrm{i}}^{\mathrm{j}}$ given by (2) for string dust with magnetic field corresponding to the line element (1) are given by

$$
\begin{aligned}
& \mathrm{T}_{1}^{1}=\frac{\mathrm{K}^{2}}{8 \pi \mathrm{A}^{2} \mathrm{~B}^{2}}=\mathrm{T}_{2}^{2} \\
& \mathrm{~T}_{3}^{3}=-\frac{\mathrm{K}^{2}}{8 \pi \mathrm{A}^{2} \mathrm{~B}^{2}}-\lambda \\
& \mathrm{T}_{4}^{4}=-\rho-\frac{\mathrm{K}^{2}}{8 \pi \mathrm{A}^{2} \mathrm{~B}^{2}}
\end{aligned}
$$

The Einstein's field equation is given by

$$
\mathrm{R}_{\mathrm{i}}^{\mathrm{j}}-\frac{1}{2} \mathrm{R} \mathrm{g}_{\mathrm{i}}^{\mathrm{j}}-\Lambda \mathrm{g}_{\mathrm{i}}^{\mathrm{j}}=-8 \pi \mathrm{T}_{\mathrm{i}}^{\mathrm{j}}
$$

(in gravitational unit $\mathrm{G}=1, \mathrm{c}=1$ )

The conservation equation

$$
\left(8 \pi T_{i}^{j}-\Lambda g_{i}^{j}\right)_{; j}=0
$$

leads to

$$
8 \pi \rho_{4}+8 \pi \rho\left(2 \frac{B_{4}}{B}+\frac{C_{4}}{C}\right)-8 \pi \lambda \frac{C_{4}}{C}+\Lambda_{4}=0 \quad\left(\sin c e \quad \frac{A_{4}}{A}=\frac{B_{4}}{B}\right)
$$

Thus the field equation (17) for the metric (1) leads to

$$
\begin{aligned}
& \frac{\mathrm{B}_{44}}{\mathrm{~B}}+\frac{\mathrm{C}_{44}}{\mathrm{C}}+\frac{\mathrm{B}_{4} \mathrm{C}_{4}}{\mathrm{BC}}=-\frac{\mathrm{K}^{2}}{\mathrm{~A}^{2} \mathrm{~B}^{2}}+\Lambda \\
& \frac{\mathrm{A}_{44}}{\mathrm{~A}}+\frac{\mathrm{C}_{44}}{\mathrm{C}}+\frac{\mathrm{A}_{4} \mathrm{C}_{4}}{\mathrm{AC}}=-\frac{\mathrm{K}^{2}}{\mathrm{~A}^{2} \mathrm{~B}^{2}}+\Lambda \\
& \frac{\mathrm{A}_{44}}{\mathrm{~A}}+\frac{\mathrm{B}_{44}}{\mathrm{~B}}+\frac{\mathrm{A}_{4} \mathrm{~B}_{4}}{\mathrm{AB}}-\frac{\alpha^{2}}{\mathrm{~A}^{2}}=\frac{\mathrm{K}^{2}}{\mathrm{~A}^{2} \mathrm{~B}^{2}}+8 \pi \lambda+\Lambda \\
& \frac{\mathrm{A}_{4} \mathrm{~B}_{4}}{\mathrm{AB}}+\frac{\mathrm{A}_{4} \mathrm{C}_{4}}{\mathrm{AC}}+\frac{\mathrm{B}_{4} \mathrm{C}_{4}}{\mathrm{BC}}-\frac{\alpha^{2}}{\mathrm{~A}^{2}}=8 \pi \rho+\frac{\mathrm{K}^{2}}{\mathrm{~A}^{2} \mathrm{~B}^{2}}+\Lambda
\end{aligned}
$$




$$
\alpha\left(\frac{\mathrm{A}_{4}}{\mathrm{~A}}-\frac{\mathrm{B}_{4}}{\mathrm{~B}}\right)=0
$$

Equation (24) leads to

$$
\mathrm{A}=\ell \mathrm{B}
$$

where $\ell$ is constant of integration.

\section{Solution of field equations}

We have equations (19) - (24) to determine A, B, C, $\lambda, \rho, \Lambda$. The solutions obtained satisfy conservation equation (19).

To get the deterministic model of the universe, we assume that shear $(\sigma)$ is proportional to expansion $(\theta)$. The motive behind assuming the condition $\sigma \alpha \theta$ is explained as :

Referring to Thorne (1967), the observations of the velocity-red shift relation for extra galactic sources suggest that the Hubble expansion of universe is isotropic within $30 \%$. More precisely, the red-shift studies place the limit $\sigma / \mathrm{H} \leq$ 0.30 where $\sigma$ is shear and $\mathrm{H}$ is the Hubble constant. Also Collins et al. (1980) have pointed out that for spatially homogeneous metric, the normal congruence to the homogeneous hyper surface satisfies the condition $\sigma / \theta$ is constant where $\theta$ is the expansion in the model. $\sigma / \theta=$ constant, leads to

$$
\mathrm{B}=\mathrm{m} \mathrm{C}^{\mathrm{n}}
$$

where $m$ and $n$ are constants. We also assume that vacuum energy density $\Lambda=\frac{a}{R^{3}}$ where $\mathrm{R}$ is scale factor. Thus, we have

$$
\Lambda=\frac{\mathrm{a}}{\mathrm{ABC}}=\frac{\mathrm{a}}{\ell \mathrm{m}^{2} \mathrm{C}^{2 \mathrm{n}+1}}
$$

Using equations (23), (24) and (25) in equation (18), we have

$$
2 \mathrm{C}_{44}+\frac{2 \mathrm{n}^{2}}{\mathrm{n}+1} \frac{\mathrm{C}_{4}^{2}}{\mathrm{C}}=-\frac{2 \mathrm{~K}^{2}}{(\mathrm{n}+1) \ell^{2} \mathrm{~m}^{4} \mathrm{C}^{4 \mathrm{n}-1}}+\frac{2 \mathrm{a}}{\ell \mathrm{m}^{2}(\mathrm{n}+1) \mathrm{C}^{2 \mathrm{n}}}
$$

Which leads to

$$
\mathrm{C}_{4}^{2}=\frac{\mathrm{K}^{2} C^{-2(2 \mathrm{n}-1)}}{\ell^{2} \mathrm{~m}^{4}\left(\mathrm{n}^{2}+n-1\right)}-\frac{2 \mathrm{a}}{(\mathrm{n}-1) \ell \mathrm{m}^{2}} C^{-(2 n-1)}+M C^{-\frac{2 n^{2}}{n+1}}
$$

To get the deterministic results, we consider two special cases:

Case (i): $n=3 / 2$

Thus, equation (29) for $n=3 / 2$ leads to

$$
\mathrm{C}_{4}^{2}=\mathrm{f}^{2}=\frac{4 \mathrm{~K}^{2}}{11 \ell^{2} \mathrm{~m}^{4}} \mathrm{C}^{-4}-\frac{4 \mathrm{a}}{\ell \mathrm{m}^{2}} \mathrm{C}^{-2}+\mathrm{MC}^{-9 / 5}
$$


Where we have assumed $\mathrm{C}_{4}=\mathrm{f}(\mathrm{C})$ and $\mathrm{M}$ is the constant of integration Thus, the metric (1) leads to

$$
\mathrm{ds}^{2}=-\left(\frac{\mathrm{dt}}{\mathrm{dC}}\right)^{2} \mathrm{~d} \mathrm{C}^{2}+\ell^{2} \mathrm{~m}^{2} \mathrm{C}^{3} \mathrm{dx^{2 }}+\mathrm{m}^{2} \mathrm{C}^{3} \mathrm{e}^{-2 \alpha x} \mathrm{dy} \mathrm{y}^{2}+\mathrm{C}^{2} \mathrm{dz}^{2}
$$

After suitable transformation of co-ordinates, the metric (29) reduces to

$$
\mathrm{ds} \mathrm{s}^{2}=-\frac{\mathrm{T}^{4} \mathrm{dT}^{2}}{\frac{4 \mathrm{~K}^{2}}{\ell^{2} \mathrm{~m}^{4}}-\frac{4 \mathrm{a}}{\ell \mathrm{m}^{2}} \mathrm{~T}^{2}+\mathrm{MT}^{11 / 5}}+\mathrm{T}^{3} \mathrm{dX} \mathrm{X}^{2}+\mathrm{T}^{3} \mathrm{e}^{-\frac{2 \alpha \mathrm{X}}{\ell \mathrm{m}}} \mathrm{dY^{2 }}+\mathrm{T}^{2} \mathrm{dZ} \mathrm{Z}^{2}
$$

where $\ell \mathrm{mx}=\mathrm{X}, \mathrm{my}=\mathrm{Y}, \mathrm{z}=\mathrm{Z}, \mathrm{C}=\mathrm{T}$ and cosmic time $\mathrm{t}$ is defined as

$$
\mathrm{t}=\int \frac{\mathrm{T}^{2} \mathrm{dT}}{\left(\frac{4 \mathrm{~K}^{2}}{11 \ell^{2} \mathrm{~m}^{4}}-\frac{4 \mathrm{aT}^{2}}{\ell \mathrm{m}^{2}}+\mathrm{MT}^{11 / 5}\right)^{1 / 2}}
$$

\section{Particular Solution}

if $\mathrm{M}=0$ then equation (30) leads to

$$
C_{4}^{2}=\alpha_{1} C^{-4}-\beta C^{-2}
$$

where

$$
\alpha_{1}=\frac{4 K^{2}}{11 \ell^{2} m^{4}}, \beta=\frac{4 a}{\ell m^{2}}
$$

From equation (34), we have

$$
\frac{C^{2} d C}{\sqrt{\gamma^{2}-C^{2}}}=\sqrt{\beta} d t
$$

Where $\frac{\alpha_{1}}{\beta}=\gamma^{2}$

Equation (36) leads to

$$
\frac{\gamma^{2}}{2} \sin ^{-1} \frac{C}{\gamma}-\frac{C}{2} \sqrt{\gamma^{2}-C^{2}}=\sqrt{\beta} t+\delta
$$

Where $\delta$ is constant of integration.

Case (ii): $n=1 / 2$

Equation (28) for $n=1 / 2$ leads to

$$
\frac{\mathrm{df}^{2}}{\mathrm{dC}}+\frac{1}{3} \frac{\mathrm{f}^{2}}{\mathrm{C}}=-\frac{4 \mathrm{~K}^{2}}{3 \ell^{2} \mathrm{~m}^{4} \mathrm{C}}+\frac{4 \mathrm{a}}{3 \ell \mathrm{m}^{2} \mathrm{C}}
$$

which leads to 


$$
\mathrm{C}_{4}^{2}=\mathrm{f}^{2}=-\frac{4 \mathrm{~K}^{2}}{\ell^{2} \mathrm{~m}^{4}}+\frac{4 \mathrm{a}}{\ell \mathrm{m}^{2}}+\mathrm{NC}^{-1 / 3}
$$

where $\mathrm{N}$ is constant of integration.

Therefore, the metric (1) leads to

$$
\mathrm{ds}^{2}=-\left(\frac{\mathrm{dt}}{\mathrm{dC}}\right)^{2} \mathrm{dC^{2 }}+\ell^{2} \mathrm{~m}^{2} \mathrm{Cdx^{2 }}+\mathrm{m}^{2} \mathrm{Ce}^{-2 \alpha x} \mathrm{dy}{ }^{2}+\mathrm{C}^{2} \mathrm{dz}^{2}
$$

which leads to

$$
\mathrm{ds}^{2}=-\frac{\tau^{1 / 3} \mathrm{~d} \tau^{2}}{\frac{4 \mathrm{a}}{\ell \mathrm{m}^{2}} \tau^{1 / 3}-\frac{4 \mathrm{~K}^{2}}{\ell^{2} \mathrm{~m}^{4}} \tau^{1 / 3}+\mathrm{N}}+\tau \mathrm{d} \mathrm{X}^{2}+\tau \mathrm{e}^{-\frac{2 \alpha}{\ell \mathrm{m}} \mathrm{X}} \mathrm{dY} \mathrm{Y}^{2}+\tau \mathrm{d} Z^{2}
$$

where cosmic time $t$ is defined as

$$
\mathrm{t}=\int \frac{\mathrm{t}^{1 / 6} \mathrm{~d} \tau}{\left(\frac{4 \mathrm{a}}{\ell \mathrm{m}^{2}} \tau^{1 / 3}-\frac{4 \mathrm{~K}^{2}}{\ell^{2} \mathrm{~m}^{4}} \tau^{1 / 3}+\mathrm{N}\right)^{1 / 2}}
$$

and

$$
\ell \mathrm{mx}=\mathrm{X}, \mathrm{my}=\mathrm{Y}, \mathrm{z}=\mathrm{Z} .
$$

\section{Special Case}

If $\mathrm{N}=0$ then equation (40) leads to

$$
f^{2}=C_{4}^{2}=\left(\frac{4 a \ell m^{2}-4 K^{2}}{\ell^{2} m^{4}}\right)
$$

Thus, we have

$$
\mathrm{C}=\mathrm{bt}+\mathrm{d}
$$

Where $b^{2}=\frac{4 a \ell m^{2}-4 K^{2}}{\ell^{2} m^{4}}, a \ell m^{2}>K^{2}$

and $\mathrm{d}$ is the constant of integration.

Therefore the metric (1) leads to the form

$$
\mathrm{ds}^{2}=-\mathrm{dt}^{2}+l^{2} \mathrm{~m}^{2}(\mathrm{bt}+\mathrm{d}) \mathrm{dx^{2 }}+\mathrm{m}^{2}(\mathrm{bt}+\mathrm{d}) \mathrm{e}^{-2 \alpha \mathrm{x}} \mathrm{dy}^{2}+(\mathrm{bt}+\mathrm{d})^{2} \mathrm{dz}^{2}
$$

\section{String dust model}

Equations (22) and (23) after using string dust condition $\rho=\lambda,($ Stachel[6]) leads to

$$
\frac{\mathrm{B}_{44}}{\mathrm{~B}}=\frac{\mathrm{B}_{4} \mathrm{C}_{4}}{\mathrm{BC}}
$$

Thus, we have 


$$
\mathrm{B}_{4}=\mathrm{LC}
$$

where $\mathrm{L}$ is constant of integration. Using (26) in (49), we have

$$
\mathrm{C}^{\mathrm{n}-2} \mathrm{dC}=\frac{\mathrm{L}}{\mathrm{mn}} \mathrm{dt}
$$

where $\mathrm{m}, \mathrm{n}$ are constants.

From equation (50), we have

$$
\mathrm{C}^{\mathrm{n}-1}=\beta \mathrm{t}+\gamma
$$

where

$$
\beta=\frac{\mathrm{L}(\mathrm{n}-1)}{\mathrm{mn}}
$$

Thus

$$
\mathrm{B}=\mathrm{mC}^{\mathrm{n}}=\mathrm{m}(\beta \mathrm{t}+\gamma)^{\frac{\mathrm{n}}{\mathrm{n}-1}}
$$

and

$$
\mathrm{A}=\ell \mathrm{B}=\ell \mathrm{m}(\beta \mathrm{t}+\gamma)^{\frac{\mathrm{n}}{\mathrm{n}-1}}
$$

Therefore, the metric (1) leads to

$d s^{2}=-d t^{2}+\ell^{2} m^{2}(\beta t+\gamma)^{\frac{2 n}{n-1}} d x^{2}+m^{2}(\beta t+\gamma)^{\frac{2 n}{n-1}} e^{-2 \alpha x} d y^{2}+(\beta t+\gamma)^{\frac{2}{n-1}} d z^{2}$

After using the transformations

$$
\begin{aligned}
& \ell \mathrm{m} \mathrm{x}=X, \mathrm{my}=\mathrm{Y}, \mathrm{z}=\mathrm{Z} \\
& \beta \mathrm{t}+\gamma=\tau,
\end{aligned}
$$

the metric (44) leads to

$$
\mathrm{ds}^{2}=-\frac{\mathrm{d} \tau^{2}}{\beta^{2}}+\tau^{\frac{2 \mathrm{n}}{\mathrm{n}-1}} \mathrm{dX} \mathrm{X}^{2}+\tau^{\frac{2 \mathrm{n}}{\mathrm{n}-1}} \mathrm{e}^{-\frac{2 \alpha \mathrm{X}}{\ell m}} \mathrm{~d} \mathrm{Y}^{2}+\tau^{\frac{2}{\mathrm{n}-1}} \mathrm{~d} \mathrm{Z}^{2}
$$

where $\mathrm{n}>1$.

\section{Physical and geometrical aspects}

The energy density $(\rho)$, the particle density $\left(\rho_{p}\right)$, the string tension density $(\lambda)$, the expansion $(\theta)$, the shear $(\sigma)$, vacuum energy density and deceleration parameter $(\mathrm{q})$ for the model (32) when $\mathrm{n}=3 / 2$, are given by

$$
8 \pi \rho=\frac{10 \mathrm{~K}^{2}}{11 \ell^{2} \mathrm{~m}^{4}} \frac{1}{\mathrm{~T}^{6}}-\frac{22 \mathrm{a}}{\ell \mathrm{m}^{2} \mathrm{~T}^{4}}-\frac{\alpha^{2}}{\ell^{2} \mathrm{~m}^{2}} \frac{1}{\mathrm{~T}^{3}}+\frac{21 \mathrm{M}}{4 \mathrm{~T}^{19 / 5}}
$$




$$
\begin{aligned}
& 8 \pi \rho_{\mathrm{p}}=\frac{30 \mathrm{~K}^{2}}{11 \ell^{2} \mathrm{~m}^{4} \mathrm{~T}^{6}}-\frac{18 \mathrm{a}}{\ell \mathrm{m}^{2} \mathrm{~T}^{4}}+\frac{42 \mathrm{M}}{10 \mathrm{~T}^{19 / 5}} \\
& 8 \pi \lambda=8 \pi \rho-8 \pi \rho_{\mathrm{p}}=-\frac{20 \mathrm{~K}^{2}}{11 \ell^{2} \mathrm{~m}^{4} \mathrm{~T}^{6}}-\frac{4 \mathrm{a}}{\ell \mathrm{m}^{2} \mathrm{~T}^{4}}+\frac{21 \mathrm{M}}{20 \mathrm{~T}^{19 / 5}}-\frac{\alpha^{2}}{\ell^{2} \mathrm{~m}^{2} \mathrm{~T}^{3}} \\
& \theta=\frac{\mathrm{A}_{4}}{\mathrm{~A}}+\frac{\mathrm{B}_{4}}{\mathrm{~B}}+\frac{\mathrm{C}_{4}}{\mathrm{C}} \\
& =4 \sqrt{\frac{4 \mathrm{~K}^{2}}{11 \ell^{2} \mathrm{~m}^{4}} \frac{1}{T^{6}}-\frac{4 \mathrm{a}}{\ell \mathrm{m}^{2}} \frac{1}{T^{4}}+\frac{\mathrm{M}}{\mathrm{T}^{19 / 5}}} \\
& \sigma=\frac{1}{\sqrt{3}}\left|\frac{\mathrm{B}_{4}}{\mathrm{~B}}-\frac{\mathrm{C}_{4}}{\mathrm{C}}\right| \quad\left(\text { since } \mathrm{B}=\mathrm{mc}^{3 / 2}\right) \\
& =\frac{1}{2 \sqrt{3}} \sqrt{\frac{4 \mathrm{~K}^{2}}{11 \ell^{2} \mathrm{~m}^{4}} \frac{1}{T^{6}}-\frac{4 \mathrm{a}}{\ell \mathrm{m}^{2}} \frac{1}{T^{4}}+\frac{\mathrm{M}}{\mathrm{T}^{19 / 5}}} \quad \text { using result (30) } \\
& \Lambda=\frac{\mathrm{a}}{\ell \mathrm{m}^{2}} \frac{1}{\mathrm{~T}^{4}} \\
& \mathrm{q}=-\frac{\mathrm{R}_{44} / \mathrm{R}}{\mathrm{R}_{4}^{2} / \mathrm{R}^{2}}<0
\end{aligned}
$$

The above mentioned quantities for the model (42) are given by

$$
\begin{aligned}
& 8 \pi \rho=\frac{4 \mathrm{a} \ell \mathrm{m}^{2}-6 \mathrm{~K}^{2}}{\ell^{2} \mathrm{~m}^{4} \tau^{2}}+\frac{5 \mathrm{~N}}{4 \tau^{7 / 3}}-\frac{\alpha^{2}}{\ell^{2} \mathrm{~m}^{2} \tau} \\
& 8 \pi \rho_{\mathrm{p}}=\frac{6 \mathrm{a} \ell \mathrm{m}^{2}-6 \mathrm{~K}^{2}}{\ell^{2} \mathrm{~m}^{4} \tau^{2}}+\frac{5 \mathrm{~N}}{3 \tau^{7 / 3}} \\
& 8 \pi \lambda=8 \pi \rho-8 \pi \rho_{\mathrm{p}}=-\frac{2 \mathrm{a} \ell \mathrm{m}^{2}}{\ell^{2} \mathrm{~m}^{4} \tau^{2}}-\frac{5 \mathrm{~N}}{12 \tau^{7 / 3}}-\frac{\alpha^{2}}{\ell^{2} \mathrm{~m}^{2} \tau} \\
& \theta=\frac{2}{\tau} \sqrt{\frac{4 \mathrm{a} \ell \mathrm{m}^{2}-4 \mathrm{~K}^{2}}{\ell^{2} \mathrm{~m}^{4}}+\frac{\mathrm{N}}{\tau^{1 / 3}}} \\
& \sigma=\frac{1}{\sqrt{3}}\left|\frac{\mathrm{B}{ }_{4}}{\mathrm{~B}}-\frac{\mathrm{C}_{4}}{\mathrm{C}}\right| \\
& =\frac{1}{2 \sqrt{3}} \frac{1}{\tau} \sqrt{\frac{4 \mathrm{a} \ell \mathrm{m}^{2}-4 \mathrm{~K}^{2}}{\ell^{2} \mathrm{~m}^{4}}+\frac{\mathrm{N}}{\tau^{1 / 3}}} \\
& \Lambda=\frac{\mathrm{a}}{\ell \mathrm{m}^{2} \tau^{2}}
\end{aligned}
$$




$$
\mathrm{q}=-\frac{\mathrm{R}_{44} / \mathrm{R}}{\mathrm{R}_{4}^{2} / \mathrm{R}^{2}}
$$

The above mentioned quantities for the model (47) are given by

$$
\begin{aligned}
& 8 \pi \rho=\left(\frac{5 b^{2}}{4}-\frac{K^{2}}{l^{2} m^{4}}-\frac{a}{l m^{2}}\right) \frac{1}{(b t+d)^{2}}-\frac{\alpha^{2}}{l^{2} m^{2}(b t+d)} \\
& 8 \pi \lambda=-\left(\frac{b^{2}}{4}+\frac{K^{2}}{l^{2} m^{4}}+\frac{a}{l m^{2}}\right) \frac{1}{(b t+d)^{2}}-\frac{\alpha^{2}}{l^{2} m^{2}(b t+d)} \\
& 8 \pi \rho_{p}=8 \pi \rho-8 \pi \lambda=\frac{3 b^{2}}{2(b t+d)^{2}} \\
& \theta=\frac{A_{4}}{A}+\frac{B_{4}}{B}+\frac{C_{4}}{C} \\
& =\frac{2 B_{4}}{B}+\frac{C_{4}}{C}=\frac{2 C_{4}}{C} \\
& =\frac{2 b}{(b t+d)} \\
& H=\frac{\theta}{3}=\frac{2 b}{3(b t+d)} \\
& \sigma=\frac{1}{\sqrt{3}}\left|\frac{B_{4}}{B}-\frac{C_{4}}{C}\right| \\
& =\frac{1}{2 \sqrt{3}}\left|\frac{b}{(b t+d)}\right| \\
& \text { as } \mathrm{B}=\mathrm{C}^{1 / 2} \\
& \mathrm{R}^{3}=\mathrm{ABC}=l \mathrm{~B}^{2} \mathrm{C}=l \mathrm{C}^{2} \\
& =l(\mathrm{bt}+\mathrm{d})^{2} \\
& q=-\frac{\frac{R_{44}}{R}}{\frac{R_{4}^{2}}{R^{2}}} \\
& =\frac{1}{2} \\
& \Lambda=\frac{a}{l m^{2}(b t+d)^{2}}
\end{aligned}
$$

The energy density $(\rho)$, the string tension density $(\lambda)$, the expansion $(\theta)$, the shear $(\sigma)$, the vacuum energy density $(\Lambda)$ for string dust model (56) are given by

$$
\begin{aligned}
\rho & =\frac{\left(\mathrm{n}^{2}+2 \mathrm{n}\right)}{(\mathrm{n}-1)^{2}} \frac{\beta^{2}}{\tau^{2}}-\frac{\alpha^{2}}{\ell^{2} \mathrm{~m}^{2} \tau^{\frac{2 \mathrm{n}}{\mathrm{n}-1}}}-\frac{\mathrm{K}^{2}}{\ell^{2} \mathrm{~m}^{4} \tau^{\frac{4 \mathrm{n}}{\mathrm{n}-1}}}-\frac{\mathrm{a}}{\ell \mathrm{m}^{2} \tau^{\frac{2 \mathrm{n}+1}{\mathrm{n}-1}}} \\
& =\lambda \\
\theta & =\frac{\mathrm{A}_{4}}{\mathrm{~A}}+\frac{\mathrm{B}_{4}}{\mathrm{~B}}+\frac{\mathrm{C}_{4}}{\mathrm{C}} \\
& =\left(\frac{2 \mathrm{n}+1}{\mathrm{n}-1}\right) \frac{\beta}{\tau}
\end{aligned}
$$




$$
\begin{aligned}
& \mathrm{H}=\frac{\theta}{3}=\left(\frac{2 \mathrm{n}+1}{\mathrm{n}-1}\right) \frac{\beta}{3 \tau} \\
& \sigma=\frac{1}{\sqrt{3}}\left(\frac{\mathrm{B}_{4}}{\mathrm{~B}}-\frac{\mathrm{C}_{4}}{\mathrm{C}}\right) \\
& =\frac{1}{\sqrt{3}} \frac{\beta}{\tau} \\
& \Lambda=\frac{\mathrm{a}}{\ell \mathrm{m}^{2} \tau^{\frac{2 \mathrm{n}+1}{\mathrm{n}-1}}}
\end{aligned}
$$

The deceleration parameter (q) for the model (45) are given by

$$
\mathrm{q}=-\frac{\mathrm{R}_{44} / \mathrm{R}}{\mathrm{R}_{4}^{2} / \mathrm{R}^{2}}
$$

where

$$
\mathrm{R}^{3}=\mathrm{ABC}=\ell \mathrm{C}^{2 \mathrm{n}+1}
$$

Thus

$$
\frac{\mathrm{R}_{4}}{\mathrm{R}}=\frac{(2 \mathrm{n}+1)}{3(\mathrm{n}-1)} \frac{\beta}{\tau}
$$

and

$$
\frac{R_{44}}{R}=-\frac{\left(2 n^{2}-7 n-4\right) \beta^{2}}{9(n-1)^{2} \tau^{2}}
$$

Thus

$$
\mathrm{q}=\frac{\mathrm{n}-4}{2 \mathrm{n}+1}
$$

\section{State finder parameters $\{\mathbf{r}, \mathbf{s}\}$}

The state finder parameters effectively differentiaties between forms of dark energy and provide a simple diagnosis whether a particular model fits into the basic observational data. Following Sahni et al. (2003), the state finder parameter for the model (56) are given by

$$
\mathrm{r}=1+\frac{3 \dot{\mathrm{H}}}{\mathrm{H}^{2}}+\frac{\ddot{\mathrm{H}}}{\mathrm{H}^{3}}=1
$$

and

$$
\mathrm{s}=\frac{\mathrm{r}-1}{3\left(\mathrm{q}-\frac{1}{2}\right)}=0
$$


where $\mathrm{H}=\frac{\beta^{2}}{\tau}$. Thus $\mathrm{r}=1, \mathrm{~s}=0$ agrees with $\Lambda \mathrm{CDM}$ model where $\beta \mathrm{t}+\gamma=\tau$ and $\beta=2 / 3$

\section{Conclusions}

The equation of state are restricted by energy conditions given by Hawking and Ellis (1974). The dominant energy conditions imply that $\rho \geq 0$ and $\rho^{2} \geq \lambda^{2}$. Now $\rho \geq 0$ for the model (32) and (42) lead to

$$
\frac{10 \mathrm{~K}^{2}}{11 \ell^{2} \mathrm{~m}^{4}} \frac{1}{\mathrm{~T}^{6}}+\frac{21 \mathrm{M}}{4 \mathrm{~T}^{19 / 5}} \geq \frac{22 \mathrm{a}}{\ell \mathrm{m}^{2} \tau^{4}}+\frac{\alpha^{2}}{\ell^{2} \mathrm{~m}^{2} \tau^{3}}
$$

and

$$
\frac{4 \mathrm{a} \ell \mathrm{m}^{2}}{\ell^{2} \mathrm{~m}^{4} \tau^{2}}+\frac{5 \mathrm{~N}}{4 \tau^{7 / 3}} \geq \frac{\alpha^{2}}{\ell^{2} \mathrm{~m}^{2} \tau}+\frac{6 \mathrm{~K}^{2}}{\ell^{2} \mathrm{~m}^{4} \tau^{2}}
$$

Both the models start with a big bang at $\mathrm{T}=0$ and $\tau=0$ respectively and expansion decreases with time. The vacuum energy density $(\Lambda) \alpha \frac{1}{T^{4}}$ for the model (32) while for the model (42), $\Lambda \alpha \frac{1}{\tau^{2}}$ which leads to the result as obtained by Beesham (1993). The expressions (58) and (66) for particle density $\left(\rho_{p}\right)$ indicate that the magnetic field is directly linked with the matter. For both the models, the weak energy conditions $\rho \geq 0, \lambda<0$ (Hawking and Ellis (1974)) are satisfied. For $\Lambda=0$, the models leads to the model obtained by Tikekar and Patel (1992) and if $\Lambda$ $=0$, (1990).

The energy condition $\rho \geq 0$ in the presence of magnetic field for the model (56) leads to

$$
\frac{\left(n^{2}+2 n\right)}{(n-1)^{2}} \frac{\beta^{2}}{\tau^{2}} \geq \frac{\alpha^{2}}{\ell^{2} m^{2} \tau^{\frac{2 n}{n-1}}}+\frac{K^{2}}{\ell^{2} m^{4} \tau^{\frac{4 n}{n-1}}}+\frac{\alpha}{\ell \tau^{\frac{2 n+1}{n-1}}}
$$

The model satisfies energy condition in presence and absence of magnetic field. The solutions so obtained satisfy conservation equation (19). (Please see appendix) Since $\frac{\sigma}{\theta} \neq 0$, hence the model does not isotropize due to the presence of string where $n \neq 1$ (Letelier (1983)). As soon as strings disappear, the anisotropy also disappear. The models isotropize at late time. The deceleration parameter $\mathrm{q}<0$ if $\mathrm{n}$ $<4$ and $\mathrm{q}>0$ if $\mathrm{n}>4$. Thus the model (56) represents decelerating and accelerating phases of universe which matches with recent astronomical observations. The vacuum energy density decreases with time. The model (56) has Point Type singularity at $\tau=0$ provided $n>1$ (MacCallum (1971)). The state 
finder parameters $\{\mathrm{r}, \mathrm{s}\}$ are in agreement for Planck (2013) results (Sahani et al.(2003)).

\section{References}

Astier, P. et al.: J. Astronomy and Astrophys. 447, 31 (2007)

B. Zel'dovich, Ya.: Sov. Phys. 11, 381 (1968)

Bali, R., Anjali: Astrophys. and Space-Sci. 302, 201 (2006)

Bali, R., Jain, S.: Astrophys. Space-Sci. 311, 401 (2007)

Bali, R., Jain, S.: Int. J. Mod. Phys. D16, 11 (2007)

Bali, R., Pareek, U.K.: Astrophys. Space-Sci. 318, 237 (2008)

Bali, R., Singh, J.P.: Int. J. Theor. Phys. 47, 3288 (2008)

Bali, R., Singh, S.: Int. J. Theor Phys. 53, 2082 (2014)

Banerjee, A., Sanyal, A.K. and Chakraborty, S.: Pramana - J. Phys. 34, 1 (1990)

Beesham, A.: Phys. Rev. D 48, 3539 (1993)

Berman, M.S.: Phys. Rev. D 43, 1075 (1990)

Bertolami, O.: Novae Cim. B 93, 36 (1986)

Bronnikov, K.A., Chudayeva, E.N. and Shikin, G.N.: Class. Quant. Gravity 21, 3389 (2004)

Chakraborty, N.C., Chakraborty, S.: Int. J. Mod. Phys. D 10, 723 (2001)

Chen, W.: and Wu, Y.S.: Phys. Rev. D 41, 695 (1990)

Collins, C.B., Glass, E.N., Wilkinson, D.N.: Gen. Relativ. Gravit. 12, 805 (1980)

Everet, A.E.: Phys. Rev. D 24, 858 (1981)

Hawking, S.W., Ellis, G.F.R.: The Large Scale Structure of Space-Time, Cambridge University Press, p.88 (1974)

Kibble, T.W.B.: J. Phys. A 9, 1387 (1976)

Lemaitre, G.: Proc. Nat. Acad. Sciences (USA), 20, 12 (1934)

Letelier, P.S.: Phys. Rev. D 20, 1294 (1979)

Letelier, P.S.: Phys. Rev. D 28, 2414 (1983)

MacCallum, M.A.H.: Comm. Math. Phys. 20, 57 (1971)

Melvin, M.A.: Anu. Newyork Acad. Sci. 262, 233 (1975)

Perlmutter, S. et al.: Astrophys. J. 517, 565 (1999)

Riess, A.G. et al.: Astron. J. 116, 1009 (1998)

Saha, B.: Int. J. Theor. Phys. 52, 1314 (2013)

Saha, B.: J. Phys. A. : Math. Theor. 40, 14011 (2007)

Sahni, V., Saini, T.D., Starobinsky, A., Alam, U.: JETP Lett. 77, 201 (2003) 
Singh, G.P., Singh, T.: Gen. Relativi. Gravit. 31, 371 (1999)

Stachel, J.: Phys. Rev. D 21, 2171 (1980)

Thorne, K.S.: Astrophys. J. 148, 51 (1967)

Tikekar, R., Patel, L.K.: Gen. Relativ. Gravit. 24, 397 (1992)

Vilenkin, A.: Phys. Rev. D 24, 2082 (1982)

Wang, P., Meng, X.H.: Class. Quant. Gravity, 22, 283 (2005)

Wang, X.X.: Chin. Phys. Lett. 23, 1702 (2006)

Zel'dovich, Ya. B.: Mon. Not. Roy. Astron Soc. 192, 663 (1980) 


\section{Appendix}

The conservation equation

$\left(8 \pi T_{i}^{j}-\Lambda g_{i}^{j}\right)_{i j}=0$

leads to

$8 \pi T_{i j i}^{j}-\left(A g_{i}^{j}\right)_{i j}=0$

This leads to

$8 \pi\left[\frac{\partial}{\partial x^{j}}\left(T_{i}^{j}\right)+T_{i}^{l} T_{j l}^{j}-T_{l}^{j} \Gamma_{i j}^{l}\right]-\Lambda_{4}=0$

Now, we have

$$
\begin{aligned}
& 8 \pi\left[\frac{\partial}{\partial t}\left(T_{4}^{4}\right)+\left[T_{1}^{1}\left(I_{12}^{2}\right)+T_{4}^{4}\left(I_{14}^{1}+\Gamma_{24}^{2}+I_{34}^{3}\right)-T_{1}^{1}\left(I_{14}^{1}\right)+T_{2}^{2}\left(I_{24}^{2}+\Gamma_{12}^{2}\right)\right.\right. \\
& \left.\left.+T_{3}^{3}\left(\Gamma_{34}^{3}\right)+T_{4}^{4}(0)\right]\right]-\Lambda_{4}=0 \\
& \Rightarrow 8 \pi\left[\frac{\partial}{\partial t}\left(-\rho-\frac{K^{2}}{8 \pi A^{2} B^{2}}\right)+\left[\frac{K^{2}}{8 \pi A^{2} B^{2}}(-\alpha)+\left(-\rho-\frac{K^{2}}{8 \pi A^{2} B^{2}}\right)\left(\frac{A_{4}}{A}+\frac{B_{4}}{B}+\frac{C_{4}}{C}\right)\right]\right. \\
& \left.-\left[\frac{K^{2}}{8 \pi A^{2} B^{2}}\left(\frac{A_{4}}{A}\right)+\frac{K^{2}}{8 \pi A^{2} B^{2}}\left(\frac{B_{4}}{B}-\alpha\right)+\left(-\frac{K^{2}}{8 \pi A^{2} B^{2}}-\lambda\right) \frac{C_{4}}{C}\right]\right]-A_{4}=0 \\
& \left(\text { since } A=l B \& \frac{A_{4}}{A}=\frac{B_{4}}{B}\right) \\
& \Rightarrow-8 \pi \rho_{4}-\frac{\partial}{\partial t}\left(\frac{K^{2}}{l^{2} B^{4}}\right)-\frac{K^{2} \alpha}{l^{2} B^{4}}-8 \pi \rho\left(\frac{2 B_{4}}{B}+\frac{C_{4}}{C}\right)-\frac{K^{2}}{l^{2} B^{4}}\left(\frac{2 B_{4}}{B}+\frac{C_{4}}{C}\right) \\
& -\frac{K^{2}}{l^{2} B^{4}}\left(\frac{2 B_{4}}{B}-\frac{C_{4}}{C}\right)+\frac{K^{2} \alpha}{l^{2} B^{4}}+8 \pi \lambda \frac{C_{4}}{C}-A_{4} \\
& -8 \pi \rho_{4}+\frac{4 K^{2}}{l^{2} B^{4}} \frac{B_{4}}{B}-8 \pi \rho\left(\frac{2 B_{4}}{B}+\frac{C_{4}}{C}\right)-\frac{4 K^{2}}{l^{2} B^{4}}\left(\frac{B_{4}}{B}\right)-\frac{K^{2}}{l^{2} B^{4}}\left(\frac{C_{4}}{C}\right)+\frac{K^{2}}{l^{2} B^{4}}\left(\frac{C_{4}}{C}\right)+8 \pi \lambda \frac{C_{4}}{C} \\
& -A_{4}=0 \\
& \Rightarrow 8 \pi \rho_{4}+8 \pi \rho\left(\frac{2 B_{4}}{B}+\frac{C_{4}}{C}\right)-8 \pi \lambda \frac{C_{4}}{C}+A_{4}=0
\end{aligned}
$$

(Note : The direction of string is assumed along $\mathrm{z}$-axis, thus $\mathrm{x}_{3} \neq 0$, is only non-zero component of string direction)

Conservation equation is satisfied for the model (32)

$$
\begin{aligned}
& 8 \pi \rho=\frac{10 K^{2}}{11 l^{2} m^{4}} T^{-6}-\frac{22 a}{l m^{2}} T^{-4}-\frac{\alpha^{2}}{l^{2} m^{2}} T^{-3}+\frac{21 M}{4} T^{-19 / 5} \\
& 8 \pi \rho_{4}=\left(-\frac{60 K^{2}}{11 l^{2} m^{4}} T^{-6}-\frac{88 a}{l m^{2}} T^{-4}+\frac{3 \alpha^{2}}{l^{2} m^{2}} T^{-3}-\frac{399}{20} T^{-19 / 5}\right) \frac{C_{4}}{C} \\
& B=m C^{n}
\end{aligned}
$$




$$
\begin{aligned}
& \frac{B_{4}}{B}=\frac{3}{2} \frac{C_{4}}{C} \text { for } n=3 / 2 \\
& \frac{2 B_{4}}{B}+\frac{C_{4}}{C}=4 \frac{C_{4}}{C} \\
& 8 \pi \lambda=-\frac{20 K^{2}}{11 l^{2} m^{4}} T^{-6}-\frac{4 a}{l m^{2}} T^{-4}-\frac{\alpha^{2}}{l^{2} m^{2}} T^{-3}+\frac{21 M}{4} T^{-19 / 5} \\
& A=\frac{a}{l m^{2}} T^{-4} \\
& A_{4}=-\frac{4 a}{l m^{2}} T^{-4} \frac{C_{4}}{C}
\end{aligned}
$$

Conservation equation is given by

$$
\begin{aligned}
& 8 \pi \rho_{4}+8 \pi \rho\left(\frac{2 B_{4}}{B}+\frac{C_{4}}{C}\right)-8 \pi \lambda \frac{C_{4}}{C}+A_{4}=0 \\
& \Rightarrow\left(-\frac{60 K^{2}}{11 l^{2} m^{4}} T^{-6}-\frac{88 a}{l m^{2}} T^{-4}+\frac{3 \alpha^{2}}{l^{2} m^{2}} T^{-3}-\frac{399}{20} T^{-19 / 5}\right) \frac{C_{4}}{C}+32 \pi \rho \frac{C_{4}}{C} \\
& -8 \pi \lambda \frac{C_{4}}{C}-\frac{4 a}{l m^{2}} T^{-4} \frac{C_{4}}{C}=0 \\
& \Rightarrow\left(-\frac{60 K^{2}}{11 l^{2} m^{4}} T^{-6}+\frac{88 a}{l m^{2}} T^{-4}+\frac{3 \alpha^{2}}{l^{2} m^{2}} T^{-3}-\frac{399}{20} T^{-19 / 5}\right) \\
& +\left(\frac{40 K^{2}}{11 l^{2} m^{4}} T^{-6}-\frac{88 a}{l m^{2}} T^{-4}-\frac{4 \alpha^{2}}{l^{2} m^{2}} T^{-3}+21 M T^{-19 / 5}\right) \\
& +\left(\frac{20 K^{2}}{11 l^{2} m^{4}} T^{-6}+\frac{4 a}{l m^{2}} T^{-4}-\frac{21 M}{20} T^{-19 / 5}+\frac{\alpha^{2}}{l^{2} m^{2}} T^{-3}\right)-\frac{4 a}{l m^{2}} T^{-4}=0 \\
& \Rightarrow\left(-\frac{60 K^{2}}{11 l^{2} m^{4}} T^{-6}+\frac{60 K^{2}}{11 l^{2} m^{4}} T^{-6}\right)+\left(\frac{92 a}{l m^{2}} T^{-4}-\frac{92 a}{l m^{2}} T^{-4}\right)+\left(\frac{4 \alpha^{2}}{l^{2} m^{2}} T^{-3}-\frac{4 \alpha^{2}}{l^{2} m^{2}} T^{-3}\right) \\
& +\left(-\frac{399}{20}-\frac{21}{20}+21\right) M T^{-19 / 5}=0
\end{aligned}
$$

Similarly conservation equation is satisfied for the model (42). 